\title{
A Note on Nilpotent Operators
}

\author{
Abhay K. Gaur \\ Department of Mathematics, Duquesne University, Pittsburgh, USA \\ Email: gaura@duq.edu
}

Received July 16, 2012; revised September 11, 2012; accepted September 19, 2012

\begin{abstract}
We find that a bounded linear operator $T$ on a complex Hilbert space $H$ satisfies the norm relation $\left\||T|^{n} a\right\|=2 q$, $n=1,2, \cdots$, for any vector $a$ in $H$ such that $q \leq\left(\|T a\|-4^{-1}\|T a\|^{2}\right) \leq 1$. A partial converse to Theorem 1 by Haagerup and Harpe in [1] is suggested. We establish an upper bound for the numerical radius of nilpotent operators.
\end{abstract}

Keywords: Numerical Range; Numerical Radius; Nilpotent Operator Weighted Shift; Eigenvalues

\section{Introduction}

The motivation for this note is provided by the results obtained in [1-4]. Let $T$ be a bounded linear operator on a complex Hilbert space $H$. The numerical range of $T$, denoted by $W(T)$, is the subset of the complex plane and

$$
W(T)=\{(T x, x): x \in H,\|x\|=1\} .
$$

The numerical radius of $T$ is defined as,

$$
w(T)=\sup \{|z|: z \in W(T)\} .
$$

The following lemma is known and is an easy consequence of the definitions involved.

Lemma 1.1. $W(T)=\sup \left\{|| z T+\bar{z} T^{*} \|:|z|=1\right\}$, where $T^{*}$ is the adjoint operator of $T$ and $\bar{z}$ is the complex conjugate of $z$.

Berger and Stampfli in [2] have proved that if $w(T) \leq 1$ and $\left\|T^{n} x\right\|=2\|x\|$, for some $n$, then $T^{n+1} x=0$. Also, they gave an example of an operator $T$ and an element $x \in H$ such that $w(T)=1$ implies that $\left\|T^{n} x\right\| \leq k\|x\|$ and $k \geq \sqrt{2}$. In Theorem 2.1, we present a different proof of their result in [2] and show that $\sqrt{2}$ is indeed the best constant.

Theorem 2.1 also generalizes the result in [4] and provides a partial converse to Theorem 1 in [1, p. 372].

Our next main result in Theorem 2.3 gives an alternative and shorter proof of Theorem 1 in [1].

Applying Lemma 2 and Proposition 2 of [1], a new result on the numerical range of nilpotent operators on $\mathrm{H}$ is obtained in Theorem 2.4. This gives a restricted version of Theorem 1 in [3].

Finally, two examples are discussed. Example 3.1 deals with the operator $T_{q}$, where 1 is not the eigenvalue of $T_{q}$ if $1<q \leq \sqrt{2}$. Example 3.3 justifies why $w\left(T_{q}\right)$ fails to increase until and unless $q \rightarrow \sqrt{2}$.

\section{Main Results}

Theorem 2.1. The following statements are true for a bounded linear operator $T$ on a Hilbert space $H$ with $w(T)=1$.

1) $\left\|T^{n} a\right\|^{2}=2 q, a \in H, n=1,2, \cdots$, such that $q \leq\|T a\|^{2}-4^{-1},\|T a\|^{4} \leq 1$.

2) If $\left\|T^{n} a\right\|=2$ for some integer $n$, then $\left\|T^{n-1} a\right\|^{2}=\cdots\|T a\|^{2}=2$ and $T^{n+1} a=0$.

3) The set $\left\{a, T a, \cdots, T^{n} a\right\}$ forms a nontrivial subspace of $T$ so that its orthogonal complement is invariant.

Proof. 1) For each real number $\alpha$ and a postive integer, $n$, let $b=\alpha_{0} a+\cdots+\alpha_{n} T^{n} a$. Then the inner product relation $|(T b, b)| \leq(b, b)$ implies that

$$
\begin{aligned}
& \left|\alpha_{0} \alpha_{1}\left\|T_{a}\right\|^{2}+\cdots+\sum_{j, k=0 ; j \neq k-1} \alpha_{j} \alpha_{k}\left(T^{j+1} a, T^{k} a\right)\right| \\
& \leq \alpha_{0}^{2}+\cdots+\sum \alpha_{j} \alpha_{k}\left(T^{j} a, T^{k} a\right)
\end{aligned}
$$

That is,

$$
\begin{aligned}
& \alpha_{0} \alpha_{1} \int_{0}^{2 \pi}\left(\mathrm{e}^{i \theta} T a, \mathrm{e}^{i \theta} T a\right)+\cdots \\
& +\sum \alpha_{j} \alpha_{k}\left(\int_{0}^{2 \pi}\left(\mathrm{e}^{(j+1) i \theta} T^{(j+1)} a, \mathrm{e}^{k i \theta} T^{k} a\right)\right) \\
& \leq \int_{0}^{2 \pi} \alpha_{0}^{2}+\cdots+\sum \alpha_{j} \alpha_{k}\left(\int_{0}^{2 \pi}\left(\mathrm{e}^{j i \theta} T^{j} a, \mathrm{e}^{k i \theta} T^{k} a\right)\right)
\end{aligned}
$$

Hence, 


$$
\begin{aligned}
& \alpha_{0} \alpha_{1}\|T a\|^{2}\left(\int_{0}^{2 \pi} \mathrm{e}^{i \theta} \mathrm{e}^{-i \theta} \mathrm{d} \theta\right)+\cdots \\
& +\sum \alpha_{j} \alpha_{k}\left(T^{j+1} a, T^{k} a\right)\left(\int_{0}^{2 \pi} \mathrm{e}^{(j+1) i \theta} \mathrm{e}^{-k i \theta} \mathrm{d} \theta\right) \\
& \leq \alpha_{0}^{2} \int_{0}^{2 \pi} \mathrm{d} \theta+\cdots+\sum_{j, k=0 ; j \neq k-1} \alpha_{j} \alpha_{k}\left(T^{j} a, T^{k} a\right)\left(\int_{0}^{2 \pi} \mathrm{e}^{j i \theta} \mathrm{e}^{-k i \theta} \mathrm{d} \theta\right)
\end{aligned}
$$

Since

$$
\int_{0}^{2 \pi} \mathrm{e}^{m i \theta} \mathrm{e}^{-n i \theta} \mathrm{d} \theta=\left\{\begin{array}{cc}
0, & m \neq n \\
2 \pi, & m=n
\end{array}\right.
$$

it follows that

$$
\begin{aligned}
& 2 \pi \alpha_{0} \alpha_{1}\|T a\|^{2}+\cdots+2 \pi \alpha_{n-1} \alpha_{n}\left\|T^{n} a\right\|^{2} \\
& \leq 2 \pi \alpha_{0}^{2}+\cdots+2 \pi \alpha_{n}^{2}\left\|T^{n} a\right\|^{2}
\end{aligned}
$$

Dividing the above inequality by $2 \pi$, we have

$$
\begin{aligned}
& \alpha_{0} \alpha_{1}\|T a\|^{2}+\cdots+\alpha_{n-1} \alpha_{n}\left\|T^{n} a\right\|^{2} \\
& \leq \alpha_{0}^{2}+\cdots+\alpha_{n}^{2}\left\|T^{n} a\right\|^{2}
\end{aligned}
$$

Let $\Gamma$ be the following block-diagonal matix of order $n$ and

$$
\Gamma=\left(\begin{array}{cccc}
1 & -2^{-1}\|T a\|^{2} & \cdots & 0 \\
-2^{-1}\|T a\|^{2} & \|T a\|^{2} & \cdots & 0 \\
\vdots & \vdots & \ddots & \vdots \\
0 & \cdots & -2^{-1}\left\|T^{n} a\right\|^{2} & \left\|T^{n} a\right\|^{2}
\end{array}\right)
$$

If $\gamma_{n}$ denotes the determinant of $\Gamma$ such that $\gamma_{0}=1$ then the value of $\gamma_{n}$ is positive because all principal minors of $\Gamma$ are nonnegative. Suppose that $\gamma_{n} \geq 0$

$$
4^{-1} \gamma_{n-2}\left(\left\|T^{n} a\right\|^{2}\right)^{2}-\gamma_{n-1}\left(\left\|T^{n} a\right\|^{2}\right)+\gamma_{n}=0
$$

We consider the following cases:

Case 1. If $\gamma_{m}>0$ for the least $m$ then $\gamma_{m+1}+4^{-1} \gamma_{m-1}\left(\left\|T^{m+1} a\right\|^{2}\right)^{2}=0$ and $\left\|T^{n} a\right\|$ converges to zero.

Case 2. Let $\gamma_{n}>0$ for all $n$. Then $\left(\gamma_{1}-2^{-1}\left\|T^{n} a\right\|^{2}\right)^{2} \geq 0$ and by induction

$$
\left(\gamma_{n-1}-2^{-1}\left\|T^{n} a\right\|^{2} \gamma_{n-2}\right)^{2} \geq 0
$$

Further, the inequality

$$
\frac{\gamma_{n-1}}{\gamma_{n-2}}-\frac{\gamma_{n}}{\gamma_{n-1}} \geq 0
$$

implies that $\frac{\gamma_{n}}{\gamma_{n-1}}$ converges to $q$ as $n$ goes to infinity for some $q \geq 0$. Therefore from Equation (2.1), $\left\|T^{n} a\right\|^{2} \rightarrow 2 q$ as $n \rightarrow \infty$. Thus $q \leq\left\|T^{n} a\right\|^{2}-4^{-1}\|T a\|^{4}$. Obviously, $q=$ 1 only if $\|T a\|^{2}=\cdots=\cdots=2$.

2) By the assumption, $\|T a\|^{2}=4$ for some positive integer $n$. Now fom Equation (2.1), we obtain:

$$
4^{-1} \gamma_{n-2}(4)^{2}-\gamma_{n-1}(4)+\gamma_{n}=0
$$

and $\gamma_{n} \geq 0$ so that $\frac{\gamma_{n-1}}{\gamma_{n-2}} \geq 1$. The equality, $1=\gamma_{n-1}=\cdots=\gamma_{2}=\gamma_{1}$ now follows from (a) and thus $\left\|T^{n-1} a\right\|^{2}=\cdots=\|T a\|^{2}=2$. Also, $\gamma_{n+1}=0$ which gives $\left\|T^{n+1} a\right\|^{2}=0$ since $\gamma_{n}=0$.

3) To prove this case, we assume that if the vector $v$ is orthogonal to the spanning set $\left\{a, T a, \cdots, T^{n} a\right\}$ then $(a, T v)=\cdots=(T a, T v)=0$. Let $b=I a+T a+\cdots+T^{n-1}+T^{n} a+\gamma v$, for $\gamma>0$. Then

$$
\begin{aligned}
& \operatorname{Re}((T b, b)) \leq(b, b) \\
& \Rightarrow \gamma \operatorname{Re}\left(\left(a+T a+\cdots+T^{n} a+T v\right)\right) \\
& \quad+\gamma^{2} \operatorname{Re}((v, T v)) \leq\|v\|^{2} \cdots r^{2} \\
& \Rightarrow \operatorname{Re}\left(\left(a+T a+\cdots+T^{n} a, T v\right)\right) \leq 0 .
\end{aligned}
$$

Hence, $(a, T v)=\cdots=\left(T^{n} a, T v\right)=0$ for $T=\mathrm{e}^{i \theta} T$ and the spanning set $\left\{a, T a, \cdots, T^{n} a\right\}$ is a non-trivial invariant subspace on $T$.

In [2, p. 1052], an example of an operator $T$ on $H$ and an element $x$ in $H$ with $w(T)=1$, is given where $\left\|T^{n} x\right\|=\sqrt{2}$. Theorem 2.1 above establishes that $\sqrt{2}$ is the best constant in this case.

Remark 2.2. An operator $A$ on $H$ is hyponormal if $\left(A^{*} A-A A^{*}\right) \geq 0$. Let $M_{n}=\left\|A^{n} a\right\|^{2}$ then $M_{n}=\left(M_{1}\right)^{n}$, if $A$ is a hyponormal operator. Hence, $M_{n}=M_{1}^{2 n}$, $n=1,2, \cdots$, and the set of vectors $a, A a, \cdots, A^{n} a$ forms a reducing subspace of $A$.

A natural connection between Feijer's inequality and the numerical radius of a nilpotent operator was estaplished by Haagerup and Harpe in [1]. They proved, using positive definite kernals, that for a bounded linear operator $T$ on a Hilbert space $H$ such that $T^{\alpha+1}=0$ and $\|T\|=1$, then $w(T) \leq \cos \frac{\pi}{d+2}$. The external operator is shown to be a truncated shift with a suitable choice of the vector in $H$. The inequality is related to a result from Feijer about trigonometric polynomials of the form $\gamma(\theta)=\sum f_{k} \mathrm{e}^{i k \theta}$ with $f_{k} \in \mathrm{C}$. Such a polynomial is positive if $\gamma(\theta) \geq 0$ for all $\theta \in \mathrm{R}$. Here, we present a 
simplified proof of Theorem 1 in [1].

Theorem 2.3. For an operator $N$ on $H$ with $\|N\| \leq 1$ and $N^{n}=0$, we have $w(N) \leq \cos \frac{\pi}{n+1}$.

Proof. We will follow the notations of Theorem 1 in [1]. Let $S$ be the operator on $\mathrm{C}^{n}$ and $\left\{e_{k}\right\}, k=1, \cdots, n$ be the basis in $\mathrm{C}$. We define the operator $S$ as follows:

$$
S_{e_{1}}=0 \text { and } S_{e_{k}}=e_{k-1} \text { for } k=2,3, \cdots, n
$$

The matrix for $S$ gives a dialation for $T$. Let $A$ be the matrix for $S$ and

$$
A=\left(\begin{array}{ccccc}
0 & 1 & 0 & \cdots & 0 \\
0 & 0 & 1 & \cdots & 0 \\
\vdots & \vdots & \vdots & \ddots & \vdots \\
0 & 0 & 0 & \cdots & 1 \\
0 & 0 & 0 & \cdots & 0
\end{array}\right)
$$

If $U$ is a unitary operator on $\mathrm{C}^{n}$ with diagonal $A=\left\{1, z, \cdots, z^{n-1}\right\}$ then $\left\|S+S^{*}\right\|=\left\|U^{*}\left(S+S^{*}\right) U\right\|$. By Lemma 1, we have:

$$
\left\|S+S^{*}\right\|=\left\|U^{*}\left(S+S^{*}\right) U\right\|=\left\|z S+\bar{z} S^{*}\right\|
$$

This helps to define the characteristic function of a contraction.

For the operator $N$ on $H$, let $\Psi=\left(I-N^{*} N\right)^{1 / 2}$ then $\Psi$ is a positive operator and $\Psi$ depends on $N$. Let the range of $\Psi$ be denoted by $R(\Psi)$. Then the tensor product, $H_{4}=R(4) \otimes \mathrm{C}^{n}$, is a Hilbert space. We define the map $F: H \rightarrow H_{\Psi}$ so that $F$ is an isometry.

For $\lambda$, let $F(N \lambda)=\sum_{k=1}^{n} \Psi N^{k} \lambda \otimes e_{k}=(I \otimes S) F(\lambda)$ where $F(\lambda)=\sum_{k=1}^{n} \Psi N^{k-1} \lambda \otimes e_{k}, I$ is the identity operator, and $(I \otimes S)$ is an operator on $H_{\Psi}$.

Therefore $w(N) \leq w(I \otimes S)$ and $F^{*}(I \otimes S) F=N$.

Now, we claim that $w(S)=w(I \otimes S)$, for we hope that $2 w(I \otimes S)=\sup \left\{\left\|z I \otimes S+\bar{z} I \otimes S^{*}\right\|:|z|=1\right\}$ By Lemma 1.1

$$
\begin{aligned}
& =\sup \left\{\left\|z S+\bar{z} S^{*}\right\|:|z|=1\right\} \\
& =2 w(S) \\
& \Rightarrow w(N)=w(I \otimes S)=w(S)
\end{aligned}
$$

That is, $w(N) \leq w(S)$.

Since $\left\|S+S^{*}\right\|=\left\|z S+\bar{z} S^{*}\right\|$, we have:

$$
2 w(S)=\left\|S+S^{*}\right\|
$$

and

$$
2 w(S)=f\left(S+S^{*}\right)
$$

where $\rho\left(S+S^{*}\right)$ is the spectral radius of $\left(S+S^{*}\right)$. By the definition of the spectral radius, we have the characteristic polynomial $f$ such that $f(x)=0$ by $[5$, p. 179 , Example 9], the roots of $f(x)$ are given by

$$
\begin{gathered}
-2 \cos \left(\frac{k \pi}{n+1}\right), k=1,2, \cdots, n \text { and } w(N) \leq w(S) \text { and } \\
2 w(S)=\sup \left|-2 \cos \left(\frac{k \pi}{n+1}\right)\right|=\cos \left(\frac{\pi}{n+1}\right) .
\end{gathered}
$$

Karaev in [3] has proved, using Theorem 1 in [1] and the Sz.-Nagy-Foias model in [6] that the numerical range $W(N)$ of an arbitrary nilpotent operator $N$ on a complex Hilbert space $H$ is an open or closed disc centered at zero with radius less than or equal to $\|N\| \cos \left(\frac{\pi}{n+1}\right)$, $n=1,2, \cdots$.

Using Theorem 2 and the assumption that $w(N)=\cos \left(\frac{\pi}{n+1}\right),\|N\|=1$, we have $W(N)$ as a closed or an open disc centered at zero with radius equal to $\cos \left(\frac{\pi}{n+1}\right)$. In fact, we have the following theorem.

Theorem 2.4. For a nilpotent operator $N$ on $H$ with $N^{n}=0, n \geq 1$ and $w(N)=\cos \left(\frac{\pi}{n+1}\right)$, the numerical range $W(N)$ is a disc centered at zero with radius $\cos \left(\frac{\pi}{n+1}\right)$.

Proof. For any $\theta$ we must claim that $\lambda \mathrm{e}^{i \theta} \in W(N)$, for $\lambda=(N Z, Z)$ and $Z$ is a vector in $\mathrm{C}^{n}$.

From [1, p. 374, Proposition 2], we have $\alpha=\beta$. Also, for some $\phi$,

$\alpha_{2_{1}}=\left(S z_{0}, z_{0}\right)=\cos \left(\frac{\pi}{n+1}\right)=\left(\mathrm{e}^{i \theta} N z, z\right)=\beta_{2_{1}}$. Now by [1] [P.375, Lemma 2], we obtain:

$$
D z_{0}=\sum_{k=0}^{n-1} c_{k} S_{z_{0}}^{k}, k=0,1, \cdots, n-1
$$

and

$$
\sum_{k=0}^{n-1} \sum_{j=0}^{n-1} \bar{c}_{k} c_{j}\left(N^{k} z, N^{j} z\right)=1=\left\|D_{z_{0}}\right\|^{2}
$$

Let $\mu=\sum_{k=0}^{n-1} c_{k} N^{k} z$. Then:

$$
\begin{aligned}
(N \mu, \mu) & =\sum_{k=0}^{n-1} \sum_{j=0}^{n-1} \bar{c}_{k} c_{j}\left(N^{k+1} z, N^{j} z\right) \\
& =\mathrm{e}^{i \theta}\left(S z_{0}, z_{0}\right)=\mathrm{e}^{i(\theta+\phi)}(N z, z)
\end{aligned}
$$

and the theorem follows from above since $\theta$ is arbitrarily chosen. 


\section{An Application}

An operator $A$ is a unilateral weghted shift if there is an orthonormal basis $\left\{e_{n}: n \geq 0\right\}$ and a sequence of scalers $\left\{\alpha_{n}\right\}$ such that $A e_{n}=\alpha_{n} e_{n}+1$ for all $n \geq 0$. It is easy to see that $A=S D$ where $S$ is the unilateral shift and $D$ is the diagonal operator with $D e_{n}=\alpha+n e_{n}$, for all $n$. Thus, $|A|=|D|$ and $|A| e_{n}=\left|\alpha_{n}\right| e_{n}$ for all $n$. So $\left\{e_{n}\right\}$ is the basis of eigenvectors for $|A|$. Also, note that $A$ is bounded if $\left\{\alpha_{n}\right\}$ is bounded.

If $A$ is a unilateral shift then $A^{*} e_{0}=0$ and

$A^{*} e_{n}=\alpha_{n-1} e_{n-1}$ for $n \geq 1$. Consequently, for a hyponormal operator $A,\left(A^{*} A-A A^{*}\right) e_{0}=\alpha_{0}^{2} e_{0} \geq 0$ and $\left(A^{*} A-A A^{*}\right) e_{n}=\left(\alpha_{n}^{2}-\alpha_{n-1}^{2}\right) e_{n}$ for $n \geq 1$. A wighted shift is hyponormal if and only if its weight sequence is increasing.

Example 3.1. Let $T_{q}$ be an operator on $H=l^{2}$ such that $T_{q} e_{n}=e_{n+1}$ and $T_{q} e_{1}=q e_{2}$ for $n>1$ and $q \geq 0$. Here, we show that 1 is not an eigenvalue of $T_{q}$ if $q \in(1, \sqrt{2}]$. We prove our claim by contradiction

Let 1 be an eigenvalue of $T_{q}$. Then, there exists $f \in H$ with $2 f_{1}=q f_{2}$ and $f_{n}+f_{n+2}^{q}=2 f_{n+1}, n=2,3, \cdots$. It is not hard to see that:

$$
f_{3}=\frac{2\left(f_{2}^{2}-f_{1}^{2}\right)}{f_{2}}=\frac{f_{1}\left(4-q^{2}\right)}{q}
$$

For $1<q \leq \sqrt{2}$, we have $f_{1} \leq f_{2} \leq \cdots$ and thus $f_{n+2} \geq f_{n+1}$, which shows that $f \in H$, contrary to our assumption. Thus, 1 is not an eigenvalue of $T_{q}$ if $q \in(1, \sqrt{2}]$.

Remark 3.2. Following [2], if $h \leq \sqrt{2}-1=0.414$ then

$$
\lim _{h \rightarrow 0.414} \frac{(1+h)^{2}}{2 \sqrt{h(h+2)}}=1
$$

Therefore, the numerical radius, $w\left(T_{q}\right)$ is equal to 1 .

The example below shows that there exists an operator $\Phi$ such that $w\left(\Phi_{q}\right) \leq 1$ for $0 \leq q \leq \sqrt{2}$.

Example 3.3. Let $A$ be a unilateral shift. If $E$ is the orthogonal projection of $H=l^{2}$ onto the spanning set of vectors $e_{1}, e_{2}, \cdots, e_{n}$, then $A=E A E$ and $A$ has the usual matrix representation. Let

$$
B=\left(\begin{array}{ccccc}
0 & \sqrt{2} & 0 & \cdots & 0 \\
\sqrt{2} & 0 & 1 & \cdots & 0 \\
0 & 1 & 0 & \cdots & 0 \\
\vdots & \vdots & \vdots & 0 & 1 \\
0 & 0 & \cdots & 1 & 0
\end{array}\right)
$$

Then the characteristic polynomial of $B$ is given by a Chebyshev polynomial $\Psi_{n}(x)$ of the first kind. Let $\Psi_{n}(x)=\cos (n \theta)$ where $x=\cos \theta$. Then:

$$
\Psi_{n+1}(x)=2 x \Psi_{n}(x)-\Psi_{n-1}(x), n \geq 1
$$

(easily proven by trigonometric identities) and $\Psi_{n}(x)$ for $n=0,1,2, \cdots$ is a linear combination of powers of $x^{k}$. Also, det $(B-x I)=x \Psi_{n}(x)-2 \Psi_{n-1}(x)$. If $\operatorname{det}(B-x I)=0$ then the roots are given by the Chebyshev polynomial of the first kind. The roots can be found by finding the eigenvalues of matrix $B$. By [2, p. 179, Example 9], the eigenvalues of $B$ are given by $\cos \left[\frac{(1+2 q) \pi}{2(n+1)}\right]$, for $q=0,1,2, \cdots, n$.

Suppose that

$$
l_{n}=\cos \left[\frac{(1+2 q) \pi}{2(n+1)}\right]
$$

then $\lim _{n \rightarrow \infty} l_{n}=1$. Hence, $w\left(\Phi_{q}\right) \leq 1$ if $q \in[0, \sqrt{2}]$.

\section{REFERENCES}

[1] U. Haagerup and P. de la Harpe, "The Numerical Radius of a Nilpotent Operator on a Hilbert Space," Proceedings of the American Mathematical Society, Vol. 115, 1999, pp. 371-379.

[2] C. A. Berger and J. G. Stampi, "Mapping Theorems for the Numerical Range," American Journal of Mathematics, Vol. 89, 1967, pp. 1047-1055.

[3] M. T. Karev, "The Numerical Range of a Nilpotent Operator on a Hilbert Space," Proceedings of the American Mathematical Society, Vol. 132, 2004, pp. 2321-2326.

[4] J. P. Williams and T. Crimmins, "On the Numerical Radius of a Linear Operator," American Mathematical Monthly, Vol. 74, No. 7, 1967, pp. 832-833. doi: $10.2307 / 2315808$

[5] J. T. Scheick, "Linear Algebra with Applications," International Series in Pure and Applied Mathematics, McGraw-Hill, New York, 1997.

[6] B. Sz.-Nagy and C. Folias, "Harmonic Analysis of Operators on a Hilbert Sapce," North Holland, Amsterdam, 1970. 